\title{
Doppler imaging of the helium-variable star a Centauri ${ }^{\star}$
}

\author{
D. A. Bohlender ${ }^{1}$, J. B. Rice ${ }^{2}$, and P. Hechler ${ }^{2}$ \\ ${ }^{1}$ National Research Council of Canada, Herzberg Institute of Astrophysics, 5071 West Saanich Road, Victoria, BC, V9E 2E7, Canada \\ e-mail: david.bohlender@nrc-cnrc.gc.ca \\ 2 Department of Physics and Astronomy, Brandon University, Brandon, MB R7A 6A9, Canada \\ e-mail: rice@brandonu.ca
}

Received 29 January 2010 / Accepted 14 June 2010

\begin{abstract}
Aims. The helium-peculiar star a Cen exhibits interesting line profile variations of elements such as iron, nitrogen and oxygen in addition to its well-known extreme helium variability. The objective of this paper is to use new high signal-to-noise, high-resolution spectra to perform a quantitative measurement of the helium, iron, nitrogen and oxygen abundances of the star and determine the relation of the concentrations of the heavier elements on the surface of the star to the helium concentration and perhaps to the magnetic field orientation.

Methods. Doppler images have been created for the elements helium, iron, nitrogen and oxygen using the programs described in earlier papers by Rice and others. An alternative surface abundance mapping code has been used to model the helium line variations after our Doppler imaging of certain individual helium lines produced mediocre results.

Results. Doppler imaging of the helium abundance of a Cen confirms the long-known existence of helium-rich and helium-poor hemispheres on the star and we measure a difference of more than two orders of magnitude in helium abundance from one side of the star to the other. Helium is overabundant by a factor of about 5 over much of the helium-rich hemisphere. Of particular note is our discovery that the helium-poor hemisphere has a very high abundance of ${ }^{3} \mathrm{He}$, approximately equal to the ${ }^{4} \mathrm{He}$ abundance. a Cen is therefore a new member of the small group of helium-3 stars and the first well-established magnetic member of the class. For the three metals investigated here, there are two strong concentrations of abundance near the equator at longitude roughly $135^{\circ}$ consistent with the positive magnetic maximum and two somewhat weaker concentrations of abundance near longitude $315^{\circ}$ on the equator near where the helium concentration is centered and roughly where the negative peak of the magnetic field would be found. Another strong concentration is found near the equator at about longitude $45^{\circ}$ and this is not explainable in terms of any simple symmetry with the helium abundance or the apparent magnetic field main polar locations.
\end{abstract}

Key words. stars: abundances - stars: chemically peculiar - stars: magnetic field - stars: individual: a Cen

\section{Introduction}

The magnetic peculiar B (Bp) star a Cen (HR 5378, HD 125823, V761 Cen) is arguably one of the most remarkable members of the Bp class. As noted by Jaschek \& Jaschek (1967) and Jaschek et al. (1968), low-dispersion spectra show that the MK type of the star, determined primarily by the strengths of the He I lines, varies between B2 V and B8 IV, while the strengths of Si II and Si III lines observed at higher dispersion remain essentially unchanged and correspond to a spectral type of B2. Norris (1968) demonstrated that at the B2 phase the helium lines are stronger than in a normal B2 V standard while near B8 the star displays a pronounced helium deficiency and all except the strongest He I lines disappear. He also found that the He I equivalent widths, $W_{\lambda}$, varied on a $8.812 \pm 0.004$ period.

After the discovery of this extreme helium variability, a Cen was studied by numerous authors for its light and colour variability, line excitation anomalies and line strength variations. Norris (1968) failed to detect a magnetic field stronger than $700 \mathrm{G}$ but a relatively weak field was tentatively detected by

* Based on observations obtained at the Canada-France-Hawaii Telescope (CFHT) which is operated by the National Research Council of Canada, The Institut National des Sciences de l'Univers of the Centre National de la Recherche Scientifique of France, and the University of Hawaii.
Wolff \& Morrison (1974) and quantitatively confirmed by Borra et al. (1983). Photometric, spectroscopic and magnetic field data all vary on the Norris (1968) period, assumed to be the rotation period of the star.

Qualitative discussions of the distribution of helium on the surface of a Cen have been given by Norris \& Baschek (1972) and Mihalas (1973). The former proposed a single helium-strong pole on the surface of a Cen while the latter looked at a number of options including the possibility that the period was twice as long as the 8.8 period determined from the star's light curve. For the single wave 8.8 period Mihalas (1973) explored two possibilities for the helium distribution: a model with two small caps of excess helium abundance with an inclination of the star of $45^{\circ}$ and magnetic obliquity $\beta$ of $45^{\circ}$, and a second model with a single cap of excess helium with an inclination of the star of $45^{\circ}$ and $\beta$ of $90^{\circ}$.

The magnetic variations of a Cen measured by Borra et al. (1983) ruled out the two-cap or symmetric model of Mihalas (1973) as they found the sinusoidal longitudinal magnetic field curve to be nearly symmetric with extrema of -470 and $+430 \mathrm{G}$. Maximum He I line strength occurs near the negative magnetic extremum. Adopting the usual Oblique Rotator Model for the star, their work then leads to the conclusion that our views of the negative and positive magnetic poles are very similar so if the inclination is not close to $90^{\circ}$, then $\beta$ must be about $90^{\circ}$. This 
then seems to be consistent with the ideas presented by Norris $\&$ Baschek (1972) and the single cap model of Mihalas (1973) where a Cen has a single helium-strong cap centered approximately $90^{\circ}$ from the rotational axis (i.e. near the negative magnetic pole) and the star has an inclination of about $45^{\circ}$.

Considering the star's unique variability and its brightness ( $V=4.401)$, it is somewhat surprising that there has been essentially no detailed investigations of a Cen published since the advent of modern solid-state detectors in the 1980's until the recent paper of Hubrig \& Gonzalez (2007). They demonstrate that the star's metallic line spectroscopic variability is quite complex and note that a characteristic of a Cen is that some elements such as oxygen have lines where the neutral line strength varies in antiphase to the singly ionized lines. They also found weak, variable high-excitation emission lines of Si II, Mn II and Fe II in the spectrum of the star but no emission in the H or He I lines. They also noted the split appearance of the Fe II and Mn II absorption line profiles, especially at the helium line strength minimum when the Fe and Mn lines are at maximum strength, and comment upon the great difficulty of creating a Doppler image given the $v \sin i$ of the star is only about $15 \mathrm{~km} \mathrm{~s}^{-1}$.

Our interest in a Cen arose from our investigation of the stratification of ${ }^{3} \mathrm{He}$ and ${ }^{4} \mathrm{He}$ in several helium-3 stars (Bohlender 2005), the apparent stratification of helium in a Cen (Leone \& Lanzafame 1997), and the suggestion of Hunger \& Groote (1999) that a Cen may be an important transitional object where the effects of fractionation of radiatively driven winds may become important. We obtained a tentative hint of the presence of ${ }^{3} \mathrm{He}$ in a Cen from a single observation of the He I $\lambda 6678$ line obtained during the course of the helium-3 star investigation and therefore in 2003 we subsequently obtained observations of several He I lines throughout the 8.8 rotation period of the star. Along with the remarkable He I line variations it was immediately obvious that the star showed pronounced variations of metal lines as later reported by Hubrig \& Gonzalez (2007). Even though the $v \sin i$ of a Cen is at the extreme low end of what is useful for Doppler imaging and our phase resolution is quite coarse, the strong spectrum variability encouraged us to try to map the surface abundance distribution for the elements He, $\mathrm{Fe}, \mathrm{N}$, and $\mathrm{O}$ to see if there were indications of axial symmetry in these distributions that would augment the impressions of the orientation of the magnetic axis gleaned from the earlier work of Borra et al. (1983).

\section{Spectroscopic data}

Spectra of a Cen were obtained with the Canada-France-Hawaii Telescope (CFHT) and the high-resolution Gecko coude spectrograph on nine consecutive nights in 2003 June. Since Gecko's mosaic grating is a single-order echellette, the spectra were obtained at four different grating rotations to obtain 75 to $125 \AA$ long spectra in regions which included the He I lines $\lambda \lambda 4437$, 4471, 6678 and 7281, as well as $\mathrm{H} \alpha$. The He I $\lambda 7281$ line has rarely been observed because of strong atmospheric contamination in this spectral region but its relative weakness, large isotope splitting, and the very high revolving power provided by Gecko in this order $(R=158000)$ makes it an ideal diagnostic for ${ }^{3} \mathrm{He}$.

The spectra were processed with IRAF $^{1}$ and included telluric line removal in the $\mathrm{H} \alpha$ and $\mathrm{He}$ I $\lambda 7281$ spectral regions

\footnotetext{
${ }^{1}$ IRAF is distributed by the National Optical Astronomy Observatory, which is operated by the Association of Universities for Research in Astronomy (AURA), Inc., under cooperative agreement with the National Science Foundation.
}

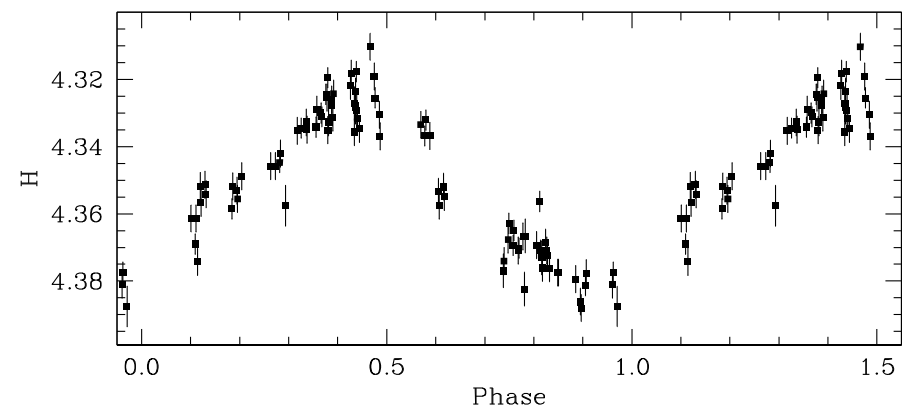

Fig. 1. Hipparcos photometry for a Cen plotted using the ephemeris given in the text. The photometric minimum in $H$ appears to be shifted by 0.05 to 0.1 cycles relative to this ephemeris defined by the $u$ light curve.

using the bright, rapidly rotating star $\alpha$ Leo as the telluric standard. Typical continuum $\mathrm{S} / \mathrm{N}$ of the individual reduced spectra is about 500 (300 for the $\lambda 7281$ spectral region) and the resolving power, as determined by the $F W H M$ of the comparison arc lines obtained before and after each grating rotation change, ranges from $93000<R<158000$.

Phases for each observation were obtained using the most recent ephemeris determined for a Cen (Catalano \& Leone 1996):

$\mathrm{JD}\left(u_{\min }\right)=2442808.376+8.817744 \pm 0.000109 \times E$.

They improved the precision of the original period determined by Norris (1968) by combining data from Norris (1971) and Pedersen \& Thomsen (1977) with new uvby photometry. While this ephemeris appears to combine data together to produce a well-defined minimum at $\phi=0$ in $u$, the helium line strength index $R$ shown in their Fig. 3 appears to indicate that the helium line strength maximum, as well as the $v b y$ photometry, precedes the $u$ photometric minimum by a small phase increment of perhaps 0.05 to 0.10 . As shown below, this is significant in that the helium maximum for our spectroscopic data appears to precede the zero-phase calculated from the above photometric ephemeris as well. In Fig. 1 we show that Hipparcos photometry of a Cen also shows the same phase shift. This difference in phase appears to be real since shifting the Hipparcos photometry to give a minimum at phase zero would require a reduction of the period by an amount approximately ten times its quoted uncertainty. There has only been about 580 and 1130 stellar rotations between the Catalano \& Leone (1996) observations, the Hipparcos observations and our spectra acquisition so the more recent data should only have errors of about 0.007 and 0.014 in phase respectively. Based on this discussion we will therefore refer to the ephemeris above as identifying the $u$ minimum.

As noted above, the negative extremum of a Cen's magnetic field occurs near the phase of maximum helium line strength or photometric minimum.

Table 1 provides a summary of our spectroscopic observations. Rotational phases are provided in both decimal and angular formats for easy comparison with our models below. A small sample of the spectra in a section of a single spectral window are presented in Fig. 2.

The number of observations and the uniformity of phase coverage is much less than ideal for Doppler imaging. However the high-resolution of the data and the surprising degree of profile shape variations apparent in Fig. 2 invites an attempt to extract some information about the distribution of elements such as He, $\mathrm{Fe}, \mathrm{N}$ and $\mathrm{O}$ over the surface in the hope that the symmetry of distribution will augment what is known about the magnetic 
Table 1. Spectroscopic observation log for a Cen.

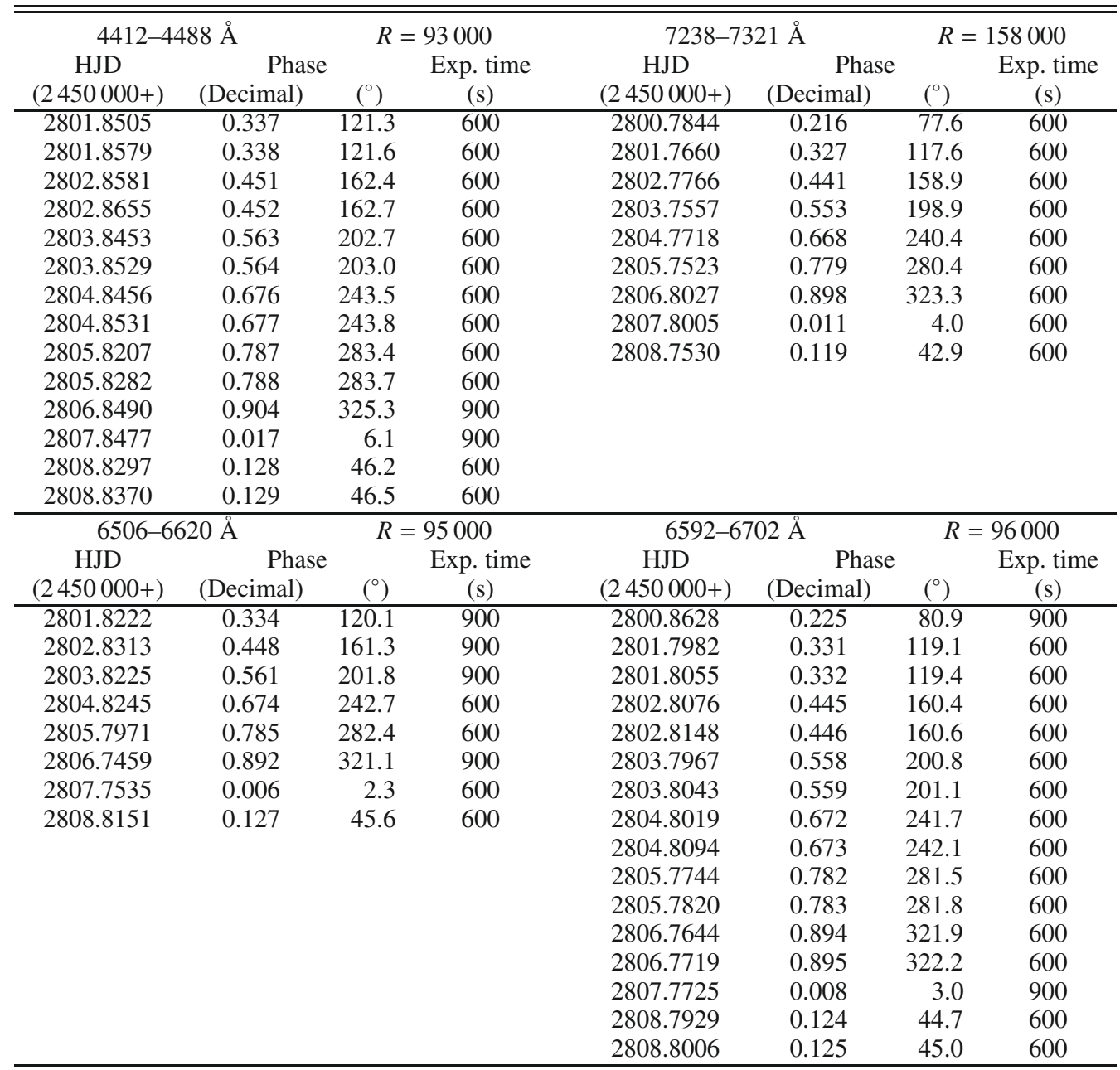

variability and perhaps indicate the orientation of the magnetic axis relative to the rotational axis.

\section{Doppler imaging}

The technique for Doppler imaging of Ap and Bp stars to obtain a map of the variable abundance of elements over their surfaces has been thoroughly covered in the literature. Examples of the computing approaches to Doppler imaging are reviewed in Rice (1996) and in Piskunov \& Rice (1993) and a comprehensive review of many of the uncertainties in Doppler imaging, although focussing in this case mainly on cool stars, is found in Rice \& Strassmeier (2000).

A critical issue for Doppler imaging is whether the same $T_{\text {eff }}$ can be assumed for the entire surface of the star or whether a variable $T_{\text {eff }}$ is required in the programming. Fortunately this issue has been reviewed in an extensive and careful analysis carried out by Molnar (1974) based on the OAO-2 ultraviolet photometry scans. Molnar concluded that neither the photometric variability nor the ultraviolet $\mathrm{Si}$ line variations were due to a variation in $T_{\text {eff }}$ over the rotation cycle of the star. Krtička et al. (2007) have also demonstrated that the light curve of the heliumstrong star HD 37776, an object quite comparable to a Cen in fundamental properties and in having quite pronounced line profile variations, can be almost completely accounted for by the inhomogeneous surface distribution of silicon and helium over the star's surface and the bound-free transitions of these elements and not surface temperature variations. More recent work on a cooler Ap star, HR 7224, also suggests that surface temperature variations do not exist in Ap stars (Krtička et al. 2009).

The $T_{\text {eff }}$ and $\log g$ values assumed for a Cen by previous authors (e.g. Molnar 1974; Norris 1971) are in the range of $19000 \mathrm{~K}$ to $20000 \mathrm{~K}$ and $\log g$ of about 4.0. We have used $T_{\text {eff }}=19000$ and $\log g=4.0$ for all models discussed in this paper.

A discussion of the adopted rotation axis inclination, $i$, is deferred until the end of this section.

\subsection{Helium}

Ideally, for a helium variable such as a Cen, it would be most informative if a Doppler image of the star's helium distribution could be obtained with at least moderate resolution. There are four He I lines in the four spectroscopic regions given in Table 1: the three singlet lines $\lambda \lambda 4437.551,6678.154$, and 7281.349, all arising from the same $2 \mathrm{p}^{1} \mathrm{P}$ lower level, and the triplet $\lambda 4471$ line and its forbidden component. The latter line is so strong and complex as to be unusable for Doppler imaging, given the relatively slow rotation of a Cen. As a first very simple step we therefore used the $4437 \AA$ line to obtain an approximate map of the helium distribution. We ignored the complex Stark broadening inherent to the line and instead used highly adjusted values for radiative, Stark and van der Waals broadening parameters of 


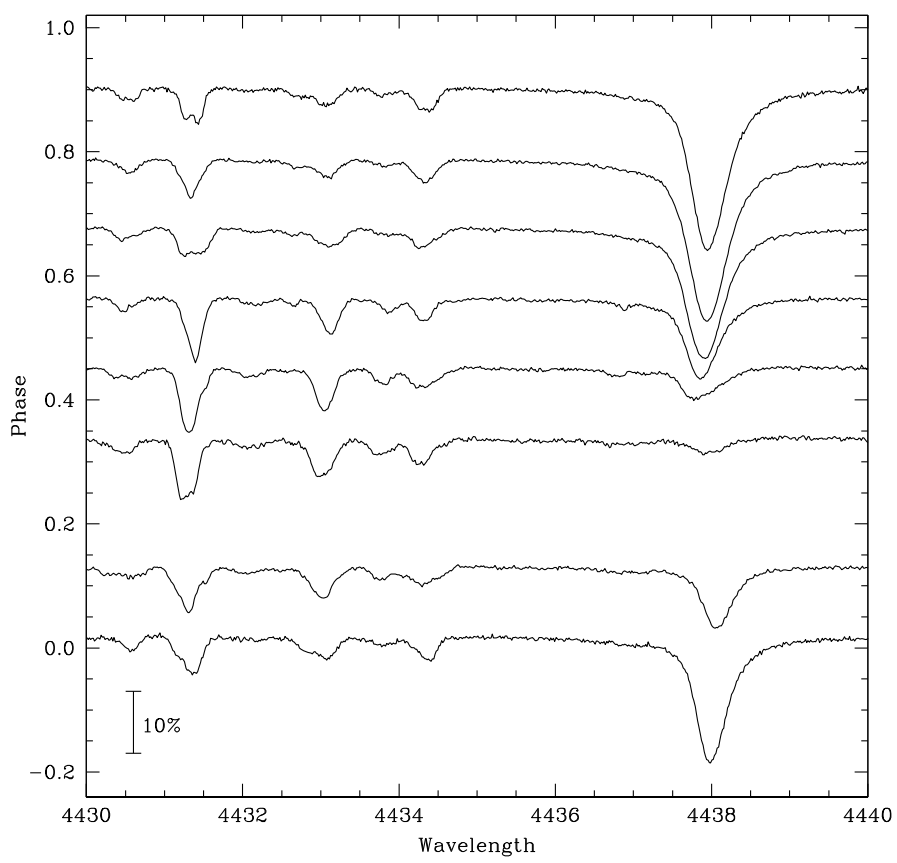

Fig. 2. A small sample of the spectra of a Cen discussed in the paper. The continuum of each spectrum is plotted at a height corresponding to the phase of the observation calculated using the ephemeris given in the text. The relative intensity scale is shown by the vertical bar at the lower left. Note the extreme change in the strength of the He I $\lambda 4437$ line as well as the pronounced profile shape variability of the metal lines.

Table 2. Lines used for Doppler imaging of a Cen and the approximate range of elemental abundances over the surface of the star.

\begin{tabular}{cccrcc}
\hline \hline $\begin{array}{c}\text { Wavelength } \\
(\AA)\end{array}$ & Ion & $\begin{array}{c}\text { E.P. } \\
(\mathrm{eV})\end{array}$ & $\log g f$ & $\begin{array}{c}\text { Min } \\
\log N / N_{\mathrm{H}}\end{array}$ & $\begin{array}{c}\operatorname{Max} \\
\log N / N_{\mathrm{H}}\end{array}$ \\
\hline 4437.551 & He I & 21.242 & -2.034 & -3.9 & -0.2 \\
6678.154 & He I & 21.242 & 0.329 & & \\
7281.349 & He I & 21.242 & -0.842 & & \\
4432.736 & N II & 23.415 & 0.580 & -4.9 & -2.9 \\
4447.030 & N II & 20.409 & 0.285 & & \\
6610.562 & N II & 21.600 & 0.433 & & \\
4414.900 & O II & 23.442 & 0.172 & -4.3 & -1.4 \\
4416.980 & O II & 23.419 & -0.077 & & \\
4419.596 & Fe III & 8.241 & -2.218 & -5.8 & -2.0 \\
4431.019 & Fe III & 8.248 & -2.572 & & \\
\hline
\end{tabular}

$6.0,-3.8$ and -6.5 respectively. Wavelengths and $\log g f$ values adopted for the helium lines (Kurucz \& Bell 1995) are provided in Table 2.

We show our Doppler image of the distribution of helium over the surface of a Cen based on the $\lambda 4437$ line in Fig. 3. Where multiple consecutive spectra were obtained we did not combine spectra; instead this provided an indication of the noise level of the spectra in the plots of line profile fits that follow. The fit to the line profiles shown in Fig. 4 is quite good and the abundance geometry is consistent with the single spot models proposed by Norris \& Baschek (1972) and Mihalas (1973): one hemisphere of the star has an enhanced abundance of helium while the other hemisphere is very helium deficient. Table 2 gives the approximate range of helium abundances determined by the Doppler imaging. These are averaged over several adjacent pixels but given the rather poor time sampling should not be interpreted too literally. When abundances are averaged over

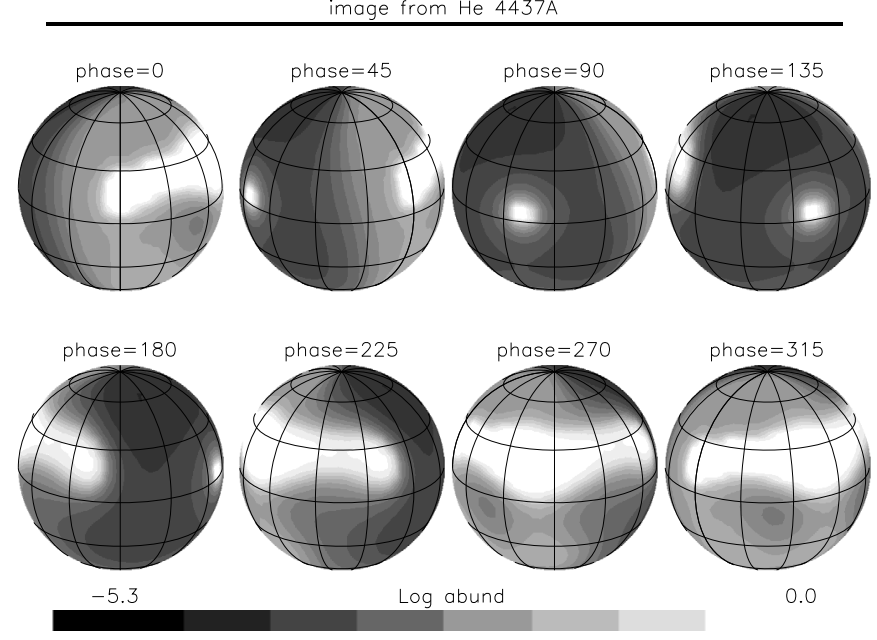

Fig. 3. An approximate map of the location of the helium concentration on a Cen obtained by a fit to the $\lambda 4437$ helium line.

the much larger obviously helium-rich and helium-difficient regions on opposite hemipheres of the star our Doppler imaging suggests that the helium abundance likely varies by a factor of 100 to 150 from one side of the star to the other.

Interestingly, we discovered some evident problems when working with the other helium lines of a Cen to produce a Doppler image of the helium surface distribution. These appear to go beyond what one might expect to have because of our neglect of Stark broadening, non-LTE affects, possible element stratification or simply a poor choice of line. In trying to invert the profiles of the $\lambda 6678$ line to form an approximate map of the helium distribution it was found that the program required an assumption of a stellar radial velocity that was $5 \mathrm{~km} \mathrm{~s}^{-1}$ more positive than was needed for the lines of the other elements as discussed in the next section. This seemed to arise because of a distortion redward of the center of the line profiles that appears roughly between phases $100^{\circ}$ and $200^{\circ}$.

Attempts to reconstruct a helium abundance map using the $\lambda 7281$ line showed a similar problem as the $\lambda 6678$ line, but even more exaggerated. The Doppler image profile fits that should match the observed profiles at phases up to about phase $100^{\circ}$ are confounded by a strong satellite feature about $0.5 \AA$ to the red that persists from about phase $100^{\circ}$ to about phase $200^{\circ}$ and then quickly and fairly completely disappears for the rest of the stellar rotation. The attempts by the program to accommodate this anomalous feature lead to persistent red-shifted distortions of the fitted profiles at other phases (see Fig. 5).

There are no known blends from lines of other elements at this wavelength but a possible solution to the poor models for the $\lambda 6678$ and $\lambda 7281$ lines is presented in Sect. 4.

\subsection{Oxygen, nitrogen and iron}

The lines of ionized oxygen and nitrogen as well as twice ionized iron listed in Table 2 were found to be the most suitable for Doppler imaging from among those available in the two observed spectroscopic regions. They presented the least problem in terms of blending issues, had an adjacent continuum that was not significantly distorted by crowding lines or the presence of broad wings of helium or hydrogen lines and were sufficiently strong to provide good line signal-to-noise. The program used for Doppler abundance imaging of Ap stars allows for blends in the calculation of the local line profiles but mapping of the 
profile fit for He $4437 \mathrm{~A}$
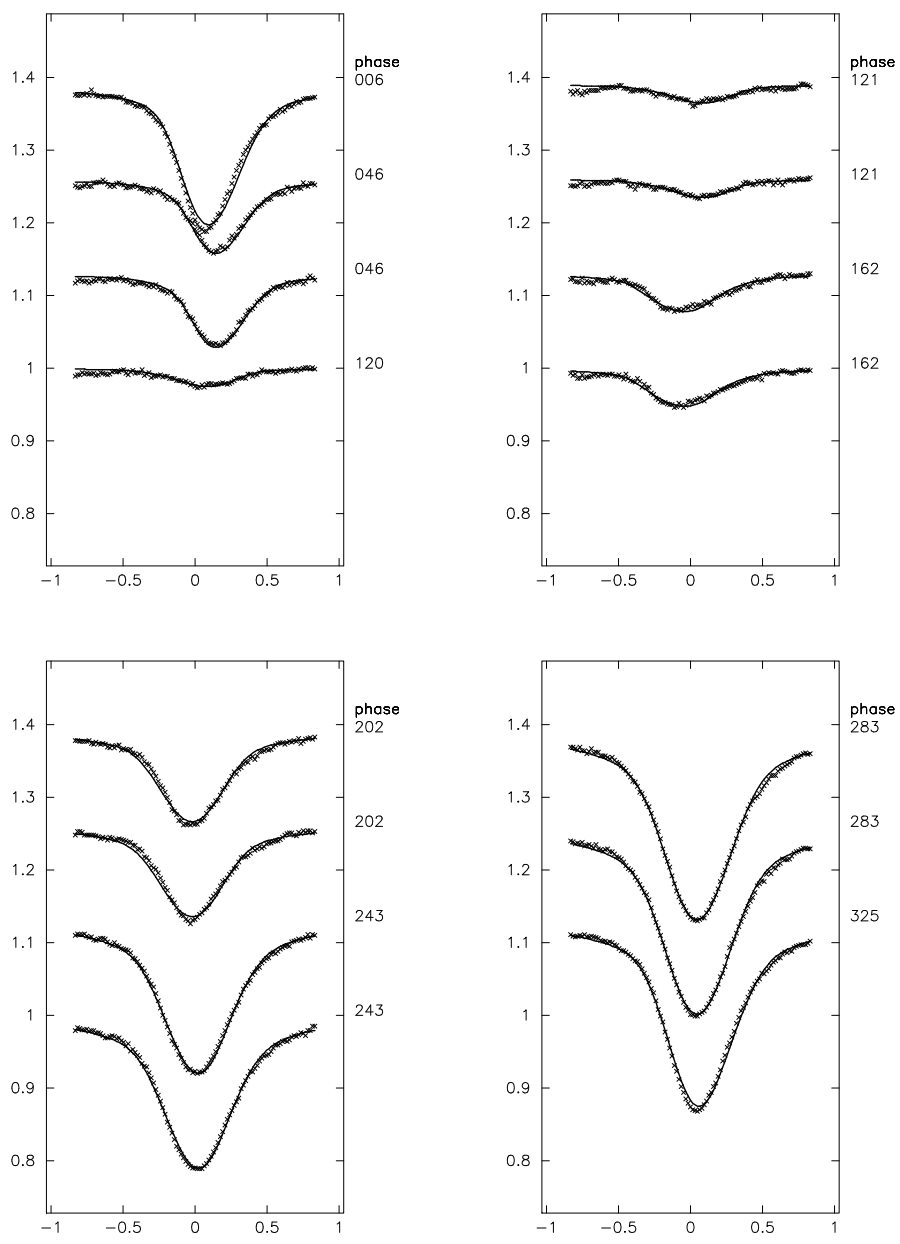

Fig. 4. He I $\lambda 4437$ line profiles variations of a Cen and the results of Doppler imaging. Observed profiles are represented by the points while the model fits are solid lines. The phases of the observed profiles are marked in degrees on the right side of the figure and the wavelength relative to line centre (in $\AA$ ) is indicated at the bottom.

abundance distribution over the stellar surface is only done for one element at a time so blends must either be of a non-variable element or blends of lines of the same element (see for example the mapping of the $7775 \AA$ line triplet of oxygen for $\epsilon$ UMa in Rice et al. 1997). The data for the lines listed in Table 2 come from the VALD database (Kupka et al. 1999).

Doppler images were generated using the lines of Table 2 for the elements iron, nitrogen and oxygen. The images were intercompared with one another and then compared with the general symmetry discussed by Mihalas (1973) and as suggested by the helium distribution as generally represented in Fig. 3.

The images developed from the Fe lines at $4419 \AA$ and $4431 \AA$ are shown as Fig. 6. The observed and model fitting to the line profiles for the $4431 \AA$ iron line are shown in Fig. 7 . The fitting to the line profiles for Fe $4419 \AA$ are very similar. The regions of enhanced iron evident on the Doppler images obtained using the two Fe lines are well defined and relatively tightly confined in area such that the five regions of peak iron abundance produce clearly identifiable dips or distortions migrating through the line profiles with the phase of the rotation of a Cen. Note the very strong similarity of the two images obtained using these two lines independently. profiles He 7281
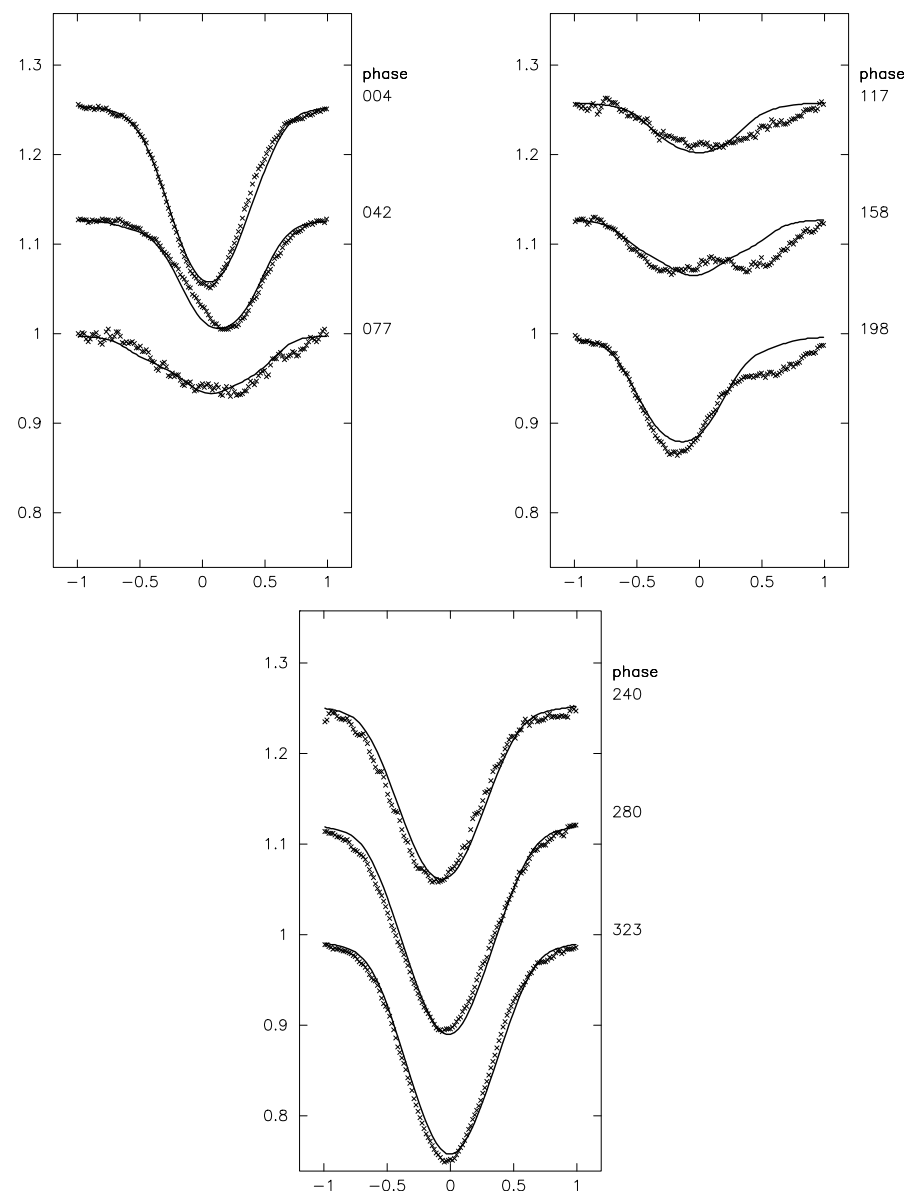

Fig. 5. As for Fig. 4 but for the He I $\lambda 7281$ line profile. Conventional Doppler Imaging is not able to reproduce the "satellite" feature longward of the line center between phases of $100^{\circ}$ and $200^{\circ}$.

The three nitrogen lines of Table 2 were each used to produce separate Doppler images using the same parameters as with the iron lines and as shown in Table 3. The line profiles for the three nitrogen lines did not show such clear and distinct distortions passing through the line profiles with rotational phase as had the two iron lines. The Doppler images from each nitrogen line then did not show such compact regions of abundance enhancement. Figure 8 shows the average of the Doppler images from the three nitrogen lines and it is evident that the image obtained for the nitrogen abundance distribution is similar to that for iron in that the placement of the abundance peaks is close to the positioning of the iron peak abundance patches but as is suggested by the less distinct pattern of line profile distortions the abundance patches are less extreme and broader in area.

Similar patterns of abundance distribution were obtained from the oxygen lines and, as with the nitrogen distributions, the pattern was generally less distinct on the surface of the star and the line profiles did not show the clear pattern of migrating dips through the line profiles with rotational phase. (Although, as with nitrogen, the line profiles exhibited profile distortions that changed with phase and that were suggestive of the migrating profile distortions seen for the iron lines.) The approximate range of abundances determined by the Doppler imaging for each element are given in Table 2.

The geometric parameters used for Doppler imaging a Cen are given in Table 3 . The choice of $70^{\circ}$ for the inclination was 

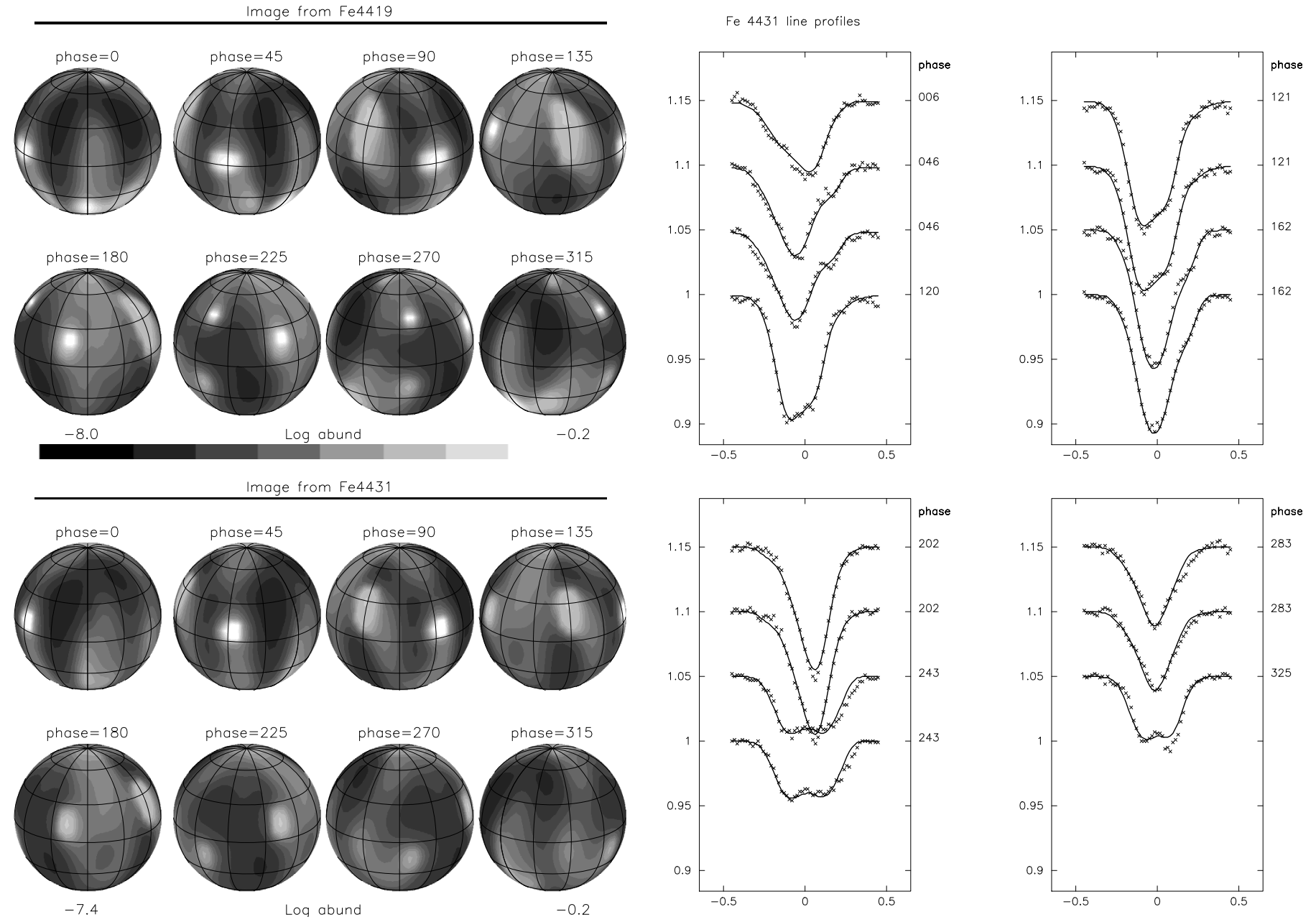

Fig. 7. As in Fig. 4 but for the line profile variations of the iron line $4431 \AA$ A.
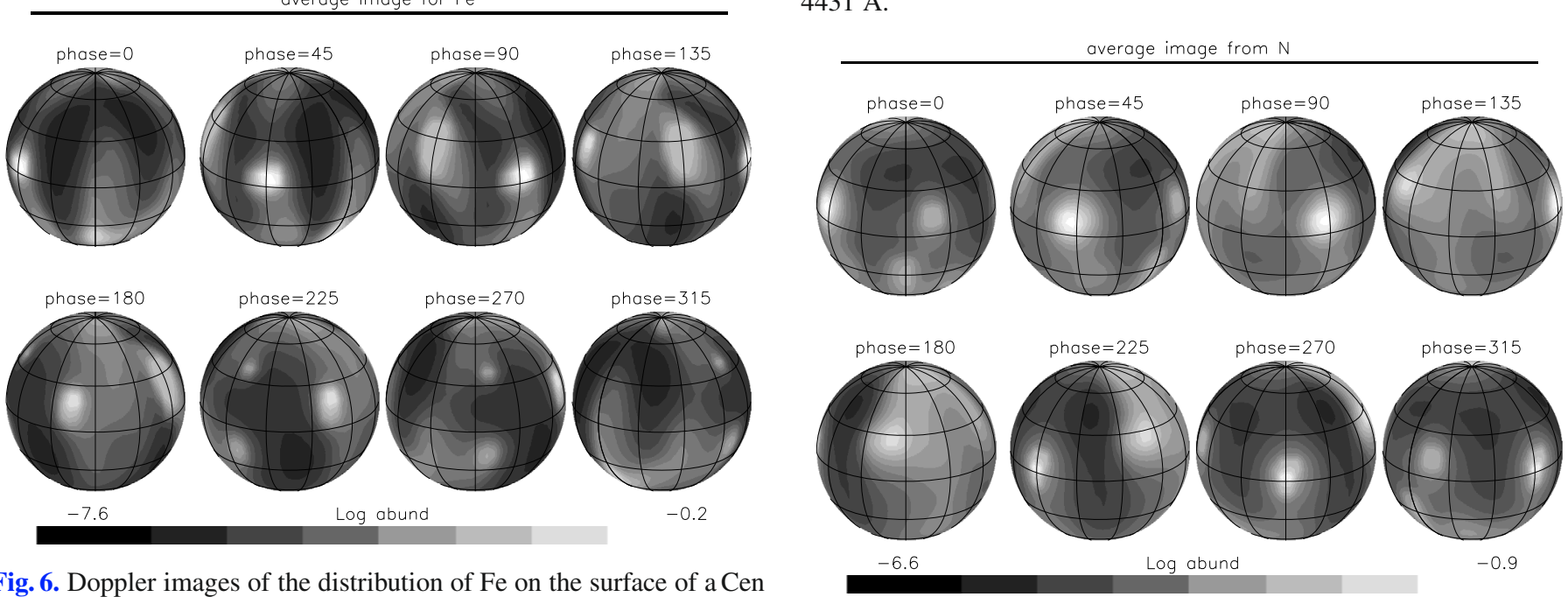

Fig. 6. Doppler images of the distribution of Fe on the surface of a Cen
reconstructed from the profile variations of the $4419 \AA$ and $4431 \AA$ Alines separately, and an image created from the combination of the Doppler images of both features.

more convoluted than in other cases of Doppler imaging. The usual preliminary search for a minimum in the error of fit of the profiles calculated from the best image formed at each of a range of inclinations to the observed profile data gave the usual broad curve with a minimum error for the iron, nitrogen and oxygen images at smaller values of inclination around $45^{\circ}$. As is also usual, the image formed from the line profiles for these elements

Fig. 8. A composite Doppler image of the distribution of nitrogen as constructed by averaging the maps reconstructed through inversion of the line profiles of the nitrogen lines listed in Table 2.

was not highly sensitive to inclination. However, the helium profiles were far better fit with an image formed using an inclination of approximately $70^{\circ}$. Exploration of the images produced and the profile fits for the $\mathrm{Fe}, \mathrm{N}$ and $\mathrm{O}$ data showed that while the overall fit to the profiles was slightly better at smaller inclinations, largely due to the better fit to the very bottom of the deeper 
Table 3. Adopted parameters for a Cen (HR 5378).

\begin{tabular}{lc}
\hline \hline Parameter & Adopted value \\
\hline$T_{\text {eff }}$ & $19000 \mathrm{~K}$ \\
$\log g$ & 4.0 \\
$v \sin i$ & $15.0 \mathrm{~km} \mathrm{~s}^{-1}$ \\
Inclination $i$ & $70^{\circ}$ \\
Rotation period & 8.817744 days \\
Radial velocity & $5.8 \pm 0.2 \mathrm{~km} \mathrm{~s}^{-1}$ \\
Micro turbulence $\xi$ & $2.5 \mathrm{~km} \mathrm{~s}^{-1}$ \\
Macro turbulence & $0.0 \mathrm{~km} \mathrm{~s}^{-1}$ \\
Radius & $2.8 \mathrm{solar}$ \\
\hline
\end{tabular}

profiles, the inverted bottom of the Fe profiles in particular at the two profiles at phase $243^{\circ}$ was far better represented in the images formed with inclination close to $70^{\circ}$. That distinctive and well-defined feature was quite poorly fit at smaller inclinations.

The choice of microturbulent velocity was made by applying the Blackwell method (Blackwell \& Shallis 1979) embedded in the SPECTRUM program (Gray \& Corbally 1994). The program was used with the nitrogen lines and suggested a microturbulence of $2.5 \mathrm{~km} \mathrm{~s}^{-1}$.

\section{Helium revisited}

Bohlender (2005) has used data identical to those presented here for a Cen to study the abundance of ${ }^{3} \mathrm{He}$ in a number of wellknown helium-3 stars (Hartoog \& Cowley 1979). In a Cen the peculiar red satellite feature in the $\lambda 7281$ line, apparent between phases $77^{\circ}$ and $240^{\circ}$, is shifted by approximately $0.5 \AA$ from the line center of the latter line. This is intriguingly close to the observed shift of $0.55 \AA$ between the ${ }^{3} \mathrm{He}$ and ${ }^{4} \mathrm{He}$ components of this line (Fred et al. 1951). A similar shift is suggested for the $\lambda 6678$ line, consistent with its isotope shift of $0.50 \AA$, but this feature is less obvious because of the greater line strength. We have therefore tried to fit the helium lines by adding a contribution from ${ }^{3} \mathrm{He}$ as described below.

First, we generated a grid of relatively simple pure hydrogen and helium non-LTE model atmospheres with $T_{\text {eff }}=19000 \mathrm{~K}$, $\log g=4.0$ and a range of $N(\mathrm{He} / \mathrm{H})$ from 0.001 to 0.8 . We used the TLUSTY program (Hubeny \& Lanz 1995) and started with the published (Lanz \& Hubeny 2007) line-blanketed model atmosphere BG19000g400v2 with $T_{\text {eff }}=19000 \mathrm{~K}, \log g=4.0$ and $N(\mathrm{He} / \mathrm{H})=0.1$ as the input atmosphere for the program. The companion spectral line synthesis program SYNSPEC was then used to produce grids of specific line and continuum intensities for the combined ${ }^{3} \mathrm{He}$ and ${ }^{4} \mathrm{He}$ lines for each helium abundance. To do so we first had to modify the version of SYNSPEC we used (v48 provided by Lanz, private communication) to incorporate broadening parameters of Dimitrijevic \& Shal-Brechot (1990) for the $\lambda 7281$ line since these were not included in the source code. SYNSPEC was also modified so that it would recognize the wavelengths of the ${ }^{3} \mathrm{He}$ components of line profiles and use the same broadening parameters as are used for the corresponding ${ }^{4} \mathrm{He}$ lines.

Unfortunately TLUSTY does not permit incorporation of separate ${ }^{3} \mathrm{He}$ and ${ }^{4} \mathrm{He}$ abundances in the production of a model atmosphere. We have therefore assumed that the helium abundance used for the TLUSTY models is the combined ${ }^{3} \mathrm{He}+{ }^{4} \mathrm{He}$ abundance and also that the lower mass of the lighter isotope has no affect on the structure of the atmosphere, or on the treatment of broadening for the lines. We then included both ${ }^{3} \mathrm{He}$ and ${ }^{4} \mathrm{He}$ line data in the input line list used by SYNSPEC and artificially

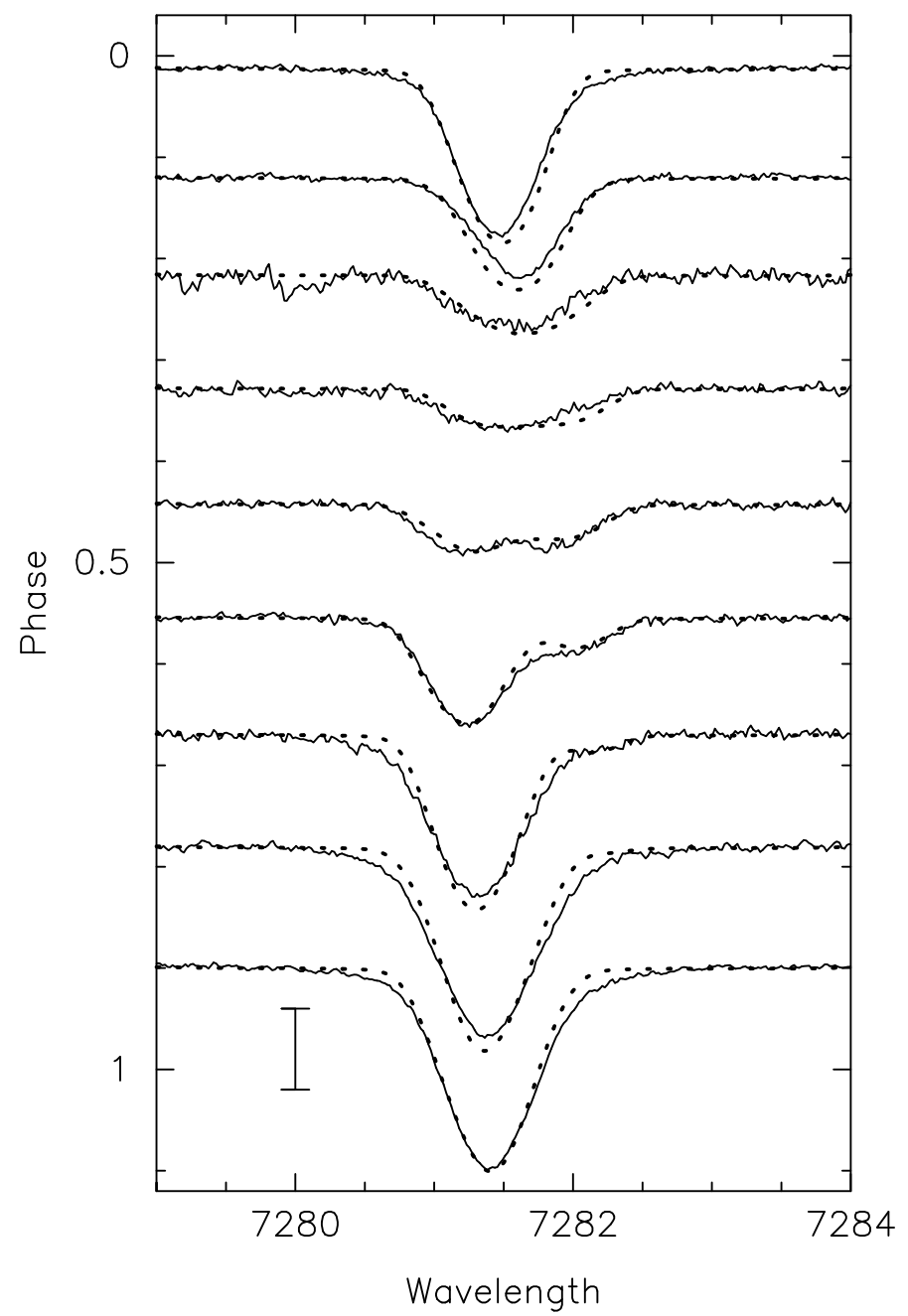

Fig. 9. Model fits for the He I $\lambda 7281$ line of a Cen in which contributions from both ${ }^{3} \mathrm{He}$ and ${ }^{4} \mathrm{He}$ isotopes are incorporated. The continuum of each spectrum is plotted at a height corresponding to the phase of the observation calculated using the ephemeris given in the text. $10 \%$ of the relatively intensity scale is shown by the vertical bar at the lower left. The helium deficient hemisphere of a Cen appears to have a huge overabundance of ${ }^{3} \mathrm{He}$.

adjusted the $\log g f$ values of the lines to set the relative abundances of the two isotopes but keep the total helium abundance used by SYNSPEC consistent with the abundance used in the model atmosphere. For example, in the case of the $\lambda 7281$ line if $N\left({ }^{3} \mathrm{He}+{ }^{4} \mathrm{He}\right)=0.1$ in the TLUSTY model and $N\left({ }^{3} \mathrm{He} /{ }^{4} \mathrm{He}\right)=1$ then identical $\log g f$ values of $(-0.842-\log 2)=-1.143$ were used for both isotopes in the input line list for SYNSPEC to provide $N\left({ }^{3} \mathrm{He} / \mathrm{H}\right)=0.05$ and $N\left({ }^{4} \mathrm{He} / \mathrm{H}\right)=0.05$. (The $\log g f$ value for the ${ }^{4} \mathrm{He} \lambda 7281$ line is -0.842 .)

The specific intensities were then used in a disk integration program that permits the placement of circular zones of different helium abundances anywhere on the disk to approximately model the surface abundance geometry. Each zone is defined by an angular radius and its colatitude and longitude relative to a point that crosses the line of sight to the observer at $\phi=0$ (see Fig. 3 of Bohlender \& Landstreet 1990, for an illustration). Bands of different abundances can also be modeled with the appropriate superposition of two circular patches. At each point of the disk integration the local helium abundance is determined and specific intensities for the appropriate line and local model atmosphere are used to construct the line profile. 
Line profiles were generated for the $\mathrm{He}$ I $\lambda \lambda 4437,4471,6678$ and 7281 lines. Fitting was conducted on a trial and error basis until reasonable fits were found for each line for as simple a configuration of abundance spots as possible. Our models indicated that a large inclination of about $70^{\circ}$ was required to reproduce the line profiles and it was this result that led us to revisit the inclination derived from Dopper imaging of the metal lines as described in the previous section. Figure 9 shows the resulting fits to the $\lambda 7281$ helium line profiles as a function of phase. While a model with just two roughly hemispherical regions with widely differing helium abundance produced a reasonably good fit, we were able to produce a substantially better fit to the line and the red satellite feature at all rotational phases by using three regions of different, but uniform, abundances. The illustrated fit is produced with a $55^{\circ}$ wide helium-rich band with $N\left({ }^{4} \mathrm{He} / \mathrm{H}\right)=0.50$ centered at a colatitude of $300^{\circ}$ and extending from $25^{\circ}$ to $80^{\circ}$ from what is assumed to be the negative magnetic pole. The $25^{\circ}$ radius polar region centered at the same colatitude has an approximately solar helium abundance of $N\left({ }^{4} \mathrm{He} / \mathrm{H}\right)=0.10$. The rest of the star's surface has $N\left(\left({ }^{3} \mathrm{He}+{ }^{4} \mathrm{He}\right) / \mathrm{H}\right)=0.004$ and $N\left({ }^{3} \mathrm{He} /{ }^{4} \mathrm{He}\right)=1$. In other words, a broad helium-rich region covers much of one hemisphere of the star, except for small region with a normal abundance near the pole, while the other hemisphere is extremely helium poor but has a huge relative overabundance of ${ }^{3} \mathrm{He}$. The ${ }^{3} \mathrm{He}$ contribution to the line profile is readily apparent between phases 0.3 and 0.7 . There is not a discernible contribution to the helium line profiles from ${ }^{3} \mathrm{He}$ in the helium-rich hemisphere. The broad helium-rich band's colatitude of $300^{\circ}$ indicates that it crosses the line of site to the observer at approximately the same phase as the Hipparcos photometric minimum discussed earlier and is also consistent with the phasing of the helium line strength photometry.

Good fits to the He I $\lambda \lambda 4437,4471$ and 6678 lines can be produced with very similar models. Minor adjustments to the helium abundances are required to improve the fits to these lines but this is not surprising. Bohlender (2005) has shown that in a few of the previously recognized helium-3 stars both isotopes of helium show evidence of abundance stratification, with ${ }^{3} \mathrm{He}$ located higher in the atmosphere than ${ }^{4} \mathrm{He}$. Our unstratified models of the $\lambda 6678$ line and especially the $\lambda 4437$ line require somewhat lower ${ }^{4} \mathrm{He}$ abundances in the helium-rich regions as well as lower ${ }^{3} \mathrm{He}$ abundances in the helium-weak hemisphere to produce satisfactory models. This is exactly what is seen in other helium-3 stars so we suggest, as did Leone \& Lanzafame (1997), that both isotopes of helium have a vertical abundance stratification in the atmosphere of a Cen. We will not pursue this question in detail until data with better phase resolution are available. More significant modifications of TLUSTY and SYNSPEC will also be needed to accommodate different vertical stratifications of both isotopes of helium in the generation of future (preferably fully-blanketed) non-LTE model atmospheres and line profiles.

Qualitatively, the overall helium abundance geometry agrees with our Doppler Imaging result: a Cen is dominated by a helium-rich region covering much of one hemisphere of the star. The small polar region with somewhat lower abundances is also obvious at phase $315^{\circ}$ in Fig. 3. However, the most remarkable result of the isotope abundance fitting is the fact that the very helium deficient hemisphere of the star has a very large relative abundance of ${ }^{3} \mathrm{He}$.

\section{Discussion}

As was discussed in the introduction to this paper, the ephemeris used here is the photometric ephemeris of Catalano \& Leone (1996) where minimum light is at phase 0 but from their Fig. 3 in their paper where they simultaneously show the light curves with the variation of the helium index $R$ it is clear that the helium maximum preceeds phase 0 by a significant fraction of a rotational cycle, perhaps of the order of 0.10 . The crude Doppler image of Fig. 3 and the profile fits shown in Fig. 9 are consistent with this as phase $315^{\circ}$ in the image appears to be roughly when the maximum helium line strength occurs and the center of the helium-rich hemisphere in our simple isotope modeling is centered at $300^{\circ}$. The minimum line strength occurs at phases of between $120^{\circ}$ and $135^{\circ}$. As conjectured by Mihalas (1973) the concentration of helium abundance is at about $90^{\circ}$ to the rotational pole (i.e. at a latitude of about $0^{\circ}$ ). The location of the maximum of helium abundance appears, from the work of Borra et al. (1983), to be close to the location of the negative magnetic pole and given the symmetry of the magnetic variation, we may assume the positive pole is close to latitude $0^{\circ}$ near a longitude (phase) of $135^{\circ}$.

We now want to compare the location of the abundance peaks that appear consistently in the multiple images derived from the separate lines of iron, oxygen and nitrogen. The location of these peaks for these elements are very similar as noted above and shown in Figs. 6 and 8. In these figures we see that there are generally five spots. A strong pair evident in the image at phase $135^{\circ}$ and just above the equator. This pair would appear to be close to the location of the magnetic positive polar region and the helium minimum but significantly these are two of the three strongest spots on the surface rather than the weakest. Further, they seem to be slightly above the equator rather than on it and they straddle the location of the polar region. This bifurcation of the peak abundance into two spots is clearly not some artifact of noise in the profile data since as has been pointed out, the individual abundance maximum regions for the iron lines are so well defined in area that they create clear dips seen migrating through the line profiles with phase (Fig. 7). Matching this pair of abundance peaks at phase $315^{\circ}$ we see a weaker pair of abundance peaks near the equator and straddling the location of the helium maximum and negative magnetic polar site.

The short description of the location of four of the five obvious abundance concentrations is that there are two stronger concentrations straddling the location of the positive magnetic polar region where the helium is weakest and two weaker concentrations straddling the location of the negative polar region where the helium is concentrated.

This leaves as a puzzle the very evident concentration near the equator at phase $45^{\circ}$ that produces probably the strongest of the features we see migrating through the iron line profiles. This feature seems totally unrelated to any of the geometry we could associate with a simple dipole-like magnetic field structure with poles located as described above or related in any way with the apparently simple asymmetric helium distribution with its concentration near phase $315^{\circ}$. If we choose to assume that abundance concentrations form where the magnetic field lines are perpendicular to the surface of the star, then it would appear that a Cen has a more complex field structure than just a simple dipole or dipole plus quadrupole pattern. The somewhat cooler helium-weak star HD 21699 is another example of a star with a single spot of enhanced helium abundance on an otherwise extremely helium-weak surface (Brown et al. 1985; Shavrina et al. 2010). It also appears to have a very peculiar magnetic field geometry (Glagolevskij \& Chuntonov 2007).

Investigations such as that by Bagnulo et al. (1999) found that while some stars can have their magnetic field modelled adequately by a dipole or dipole plus quadrupole field, most of their sample required a more complex field. In the particular case of 
the star 53 Cam with its relatively slow rotation and strong magnetic field it was seen (Kochukhov et al. 2004) that the field was quite complex. A future attempt at producing a Zeeman Doppler image of a Cen will be a challenge, but would be of considerable interest since the abundance distribution of the lighter elements examined here suggests that the magnetic field may deviate considerably from a simple structure. We encourage new magnetic field measurements of any kind for the the star to greatly improve our understanding of the abundance and magnetic field geometry of the star and the relationship between the two.

Once more detailed surface abundance and magnetic field geometries are known it would clearly also be of interest to determine if the relatively large $(\approx 0.06 \mathrm{mag})$ photometric variations observed in the star (Fig. 1) can be reproduced solely from the non-uniform abundances of the star, and in particular the extraordinary helium variability. As an aside, we point out that despite the very large variation in helium abundance over the surface of a Cen there is no discernible variability observed in the wings of the $\mathrm{H} \alpha$ profile of the star during a single rotation. This already suggests that minimal temperature or surface gravity variations are present over the surface of the star. Only in the few $\AA$ at the core of $\mathrm{H} \alpha$ are profile variations of any kind seen. These closely reflect the amplitude and shape of the variations seen in the $\mathrm{O}, \mathrm{N}$, and $\mathrm{Fe}$ lines presented here near the same rotation phase and therefore likely arise from small atmospheric structure variations caused by the non-uniform metal abundances rather than variations in the helium abundance, temperature or gravity.

Our discovery of ${ }^{3} \mathrm{He}$ in the atmosphere of a Cen adds a new member to the small group of helium-3 stars. We believe this work also makes it the first firmly established magnetic member of this class of objects; $\alpha \mathrm{Scl}$ and HR 7129 are other magnetic candidates (Hartoog \& Cowley 1979) that we feel require confirmation of their ${ }^{3} \mathrm{He}$ content with modern data. As we have already mentioned, Hunger \& Groote (1999) suggested that a Cen might represent an important transitional object among the magnetic helium-peculiar stars where the effects of fractionation of radiatively driven winds may become important. Our discovery that the star is a member of the helium-3 class of stars confirms this idea as does the suspected vertical stratification of helium in its atmosphere reported by Leone \& Lanzafame (1997) and supported by our data. It is interesting to note the fact that a Cen has a $T_{\text {eff }}$ and $\log g$ very close to the well-established, but non-variable and non-magnetic prototype of this class of stars, 3 Cen A. Clearly the atmospheres and winds of slowly-rotating peculiar stars in this mass range, whether magnetic or not, are finely tuned to enable the separation of helium isotopes in their atmosphere by either mass fractionation (Vauclair 1975) or lightinduced drift (Leblanc \& Michaud 1993).

We have already noted the discovery of weak metallic emission lines in a Cen by Hubrig \& Gonzalez (2007); the incidence of this phenomenon appears to be almost universal among the helium-3 stars.

\section{Conclusions}

Doppler images of the abundance distribution of the elements helium, iron, oxygen and nitrogen in the helium-variable star a Cen appear to be consistent with the general geometry of the abundance distribution of helium proposed by Mihalas (1973) where the location of the abundance concentrations is roughly at the rotational equator. The helium concentration is centred at a longitude between $300^{\circ}$ and $315^{\circ}$ using the ephemeris of Catalano \& Leone (1996) and we are able to reproduce abundance variations most consistently with a relatively large value of $70^{\circ}$ for the inclination of the star. For the three metallic species investigated there are two strong concentrations of abundance near the equator at longitude roughly $135^{\circ}$, consistent with the positive magnetic maximum, and two somewhat weaker concentrations of abundance near longitude $315^{\circ}$ on the equator where the helium is concentrated and roughly where the negative peak of the magnetic field would be found. Another strong concentration is found near the equator at about longitude $45^{\circ}$ and this is not explainable in terms of any simple symmetry with the helium abundance or the apparent magnetic field main polar locations. We conclude that the surface magnetic field geometry of a Cen is likely more complex that that of a simple dipole or dipole plus quadrupole.

Our detailed analysis of the variations of a number of helium line profiles show that the helium abundance of a Cen varies by a factor of approximately 125 from one hemisphere to the other if suspected vertical stratification of helium in the star's atmosphere is ignored. A large portion of one hemisphere is helium rich, except very close to the negative magnetic pole, and the other hemisphere is very helium deficient. The latter region is also found to have an extreme overabundance of ${ }^{3} \mathrm{He}$, with $N\left({ }^{3} \mathrm{He} /{ }^{4} \mathrm{He}\right) \approx 1$. As a result, a Cen is a new member of the helium-3 class of stars and the first convincing example of a magnetic member of this small group of peculiar objects.

Acknowledgements. J.B.R. thanks the Natural Sciences and Engineering Research Council of Canada for their financial support of this work. The authors thank the referee, Franco Leone, for a careful review of the manuscript.

\section{References}

Bagnulo, S., Landolfi, M., \& Landi Degl'Innocenti, M. 1999, A\&A, 343, 865 Blackwell, D. E., \& Shallis, M. J. 1979, MNRAS, 186, 673

Bohlender, D. A. 2005, EAS Publ. Ser., 17, 83

Bohlender, D. A., \& Landstreet, J. D. 1990, ApJ, 358, 274

Borra, E. F., Landstreet, J. D., \& Thompson, I. 1983, ApJS, 53, 151

Brown, D. N., Shore, S. N., \& Sonneborn, G. 1985, AJ, 90, 1354

Catalano, F. A., \& Leone, F. 1996, A\&A, 311, 230

Dimitrijevic, M. S., \& Sahal-Brechot, S. 1990, A\&AS, 82, 519

Fred, M., Tomkins, F. S., Brody, J. K., \& Hamermesh, M. 1951, Phys. Rev., 82, 406

Glagolevskij, Y. V., \& Chuntonov, G. A. 2007, Astrophysics, 50, 362

Gray, R. O., \& Corbally, C. J. 1994, AJ, 107, 742

Hartoog, M. R., \& Cowley, A. P. 1979, ApJ, 228, 229

Hubeny, I., \& Lanz, T. 1995, ApJ, 439, 875

Hubrig, S., \& Gonzalez, J. F. 2007, A\&A, 466, 1083

Hunger, K., \& Groote, D. 1999, A\&A, 351, 554

Jaschek, C., \& Jaschek, M. 1967, PASP, 70, 667

Jaschek, C., Jaschek, M., Morgan, W. W., \& Slettebak, A. 1968, AJ, 153, 873

Kochukhov, O., Bagnulo, S., Wade, G. A., et al. 2004, A\&A, 414, 613

Krtička, J., Mikulášek, Z., Zverko, J., \& Zižn̆ovský, J. 2007, A\&A, 470, 1089

Krtička, J., Mikulášek, Z., Henry, G. W., et al. 2009, A\&A, 499, 567

Kupka, F., Piskunov, N. E., Ryabchikova, T. A., Stempels H. C., \& Weiss W. W. 1999, A\&AS, 138, 119

Kurucz, R. L., \& Bell, B. 1995, Atomic Line Data, Kurucz CD-ROM No. 23

Lanz, T., \& Hubeny, I. 2007, ApJS, 169, 83

LeBlanc, F., \& Michaud, G. 1993, ApJ, 408, 251

Leone, F., \& Lanzafame, A. C. 1997, A\&A, 320, 893

Mihalas, D. 1973, ApJ, 184, 851

Molnar, M. R. 1974, ApJ, 187, 531

Norris, J. 1968, Nature, 219, 1342

Norris, J. 1971, ApJS, 23, 235

Norris, J., \& Baschek, B. 1972, A\&A, 21, 385

Pedersen, H., \& Thomsen, B. 1977, A\&AS, 30, 11

Piskunov, N. E., \& Rice, J. B. 1993, PASP, 105, 1415

Rice, J. B. 1996, in Stellar Surface Structure, ed. K.G. Strassmeier, \& J.L. Kluwer, IAU Symp., 176, 19

Rice, J. B., \& Strassmeier, K. G. 2000, A\&AS, 147, 151

Rice, J. B., Wehlau, W. H., \& Holmgren, D. E. 1997, A\&A, 326, 988

Shavrina, A. V., Glagolevskij, Yu. V., Silvester, J., et al. 2010, MNRAS, 401, 1882

Vauclair, S. 1975, A\&A, 45, 233

Wolff, S. C., \& Morrison, N. D. 1974, PASP, 86, 935 